\title{
The Structure of Pairing Strategies for $k$-in-a-row Type Games
}

\author{
Lajos Győrffy, András London, and Géza Makay ${ }^{c}$
}

\begin{abstract}
In Maker-Breaker positional games two players, Maker and Breaker, play on a finite or infinite board with the goal of claiming or preventing the opponent from getting a finite winning set, respectively. For different games there are several winning strategies for Maker or Breaker. One class of winning strategies is the so-called pairing (paving) strategies. Here, we describe all possible pairing strategies for the 9-in-a-row game. Furthermore, we define a graph of the pairings, containing 194,543 vertices and 532,107 edges, in order to give them a structure them. A complete characterization of the graph is also given.
\end{abstract}

Keywords: positional games, pairing strategies, hypergraphs

\section{Introduction}

The positional game $k$-in-a-row is played by two players on an infinite (chess)board. In the usual version of the game, the players alternately place their own marks (crosses or cirles) on previously unmarked squares, and whoever gets a winning set ( $k$-consecutive squares horizontally, vertically or diagonally) first with his own marks, wins.

In the Maker-Breaker (M-B) version of the game, Maker is the one who tries to occupy a winning set, while Breaker tries to prevent Maker from winning. There is a connection between the normal (Maker-Maker) and the M-B versions of a game. If the first player wins the normal game, she wins the M-B one as well. If Breaker wins the M-B game, then the second player can obtain a draw in a normal game. However, the converse statements are not true (see, for instance, the Tic-Tac-Toe game). The M-B version is easier for Maker because she does not need to frustrate Breaker's moves. For a comprehensive book on Maker-Breaker positional games, see e. g. Beck [2]. For different values of $k$, there are several winning strategies either for Maker or for Breaker. One group of these are the pairing (paving) strategies.

\footnotetext{
${ }^{a}$ University of Szeged, Bolyai Institute, E-mail: lgyorffy@math.u-szeged.hu

${ }^{b}$ University of Szeged, Institute of Informatics, E-mail: Iondon@inf .u-szeged.hu

${ }^{c}$ University of Szeged, Bolyai Institute, E-mail: makayg@math.u-szeged.hu
} 
A pairing strategy generally means that the possible moves of a game are paired up; if one player plays one, the other player plays its pair (see [7]). A winning pairing strategy of Breaker in the $k$-in-a-row type games is a pairing of the squares of the board such that each winning set contains at least one pair. Using the abovementioned pairing strategy, Breaker takes at least one square from each winning set, and wins the game. If there is a winning pairing strategy for Breaker in a game, then we say that there is a good pairing for the game. It was shown in $[2,3]$ (and it is illustrated in Figure 1) that there is a good pairing for the $k$-in-a-row game, if $k \geq 9$.

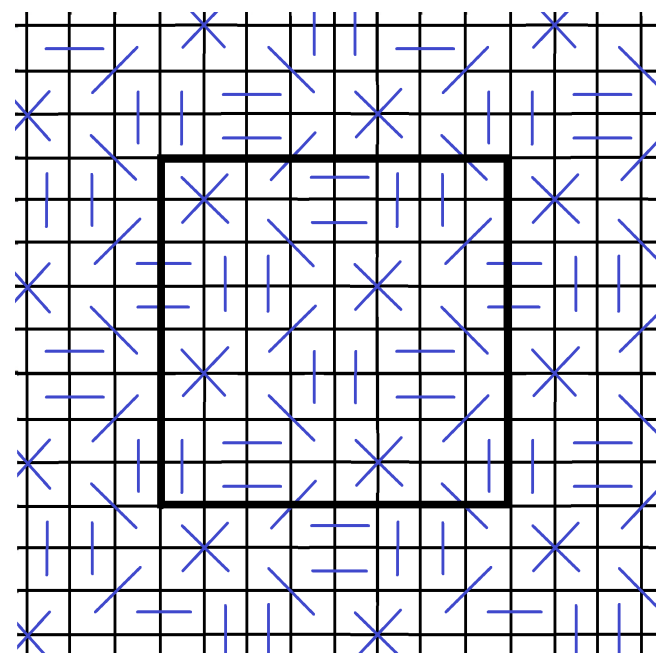

Figure 1: Hales-Jewett pairing blocks the 9-in-a-row

However, the best bound for Breaker's win was given by A. Brouwer, under the pseudonym T.G.L. Zetters [5]. He showed that Breaker will win the 8-in-a-row by tiling the board into smaller sub-boards. The best bound for Maker's win was given by Allis et al. [1]. They showed that the first player wins the classical version of the game on the $15 \times 15$ table, which implies that Maker (following first player's winning strategy from the normal game on the $15 \times 15$ table) wins the 5 -in-a-row M-B game on the infinite board, but the normal (Maker-Maker) game is still open there. The cases $k=6,7$ are also open.

In the case of $k=9$-which is the main subject of this paper-there are only highly symmetric (8- or 16-toric) good domino pairings of the board (see Györffy et al. [6]). An 8 -toric pairing means that the pairing is an extension of an $8 \times 8$ square, while domino pairing means that all pairs consist of only neighboring (vertically, horizontally or diagonally) squares called dominoes. We know from [6] that all 16-toric good pairings can be deduced from two (or more) different 8-toric pairings.

After discussing the preliminaries, in Section 3 we will define a related game, namely the $8 \times 8$ torus game. An 8 -toric good pairing for the 9 -in-a-row is also a good pairing for the $8 \times 8$ torus game, but the reverse is not true. In Section 4 , 
we introduce a program that lists all (194543) 8-toric good pairings. In Section 5, we reveal a connection between the pairings, namely two pairings are neighboring if we can obtain them from each other by a natural method. With this connection, we create a graph on the pairings. In Section 6, we analyze the network of pairings, which turns out to be a huge and sparse graph (194543 nodes and 532107 edges).

After, we investigate another similar game, namely the $k$-in-a-row game on the hexagonal board. For this game, Breaker has winning pairing strategies if $k \geq 7$. For $k=7$ we can list the 26 different 6 -toric pairings which give us a similar but smaller graph of pairings than before.

\section{Preliminaries}

\subsection{Pairing strategies}

Given a hypergraph $\mathcal{H}=(V, E)$, where $V=V(\mathcal{H})$ and $E=E(\mathcal{H}) \subseteq \mathcal{P}(\mathcal{H})=\{S$ : $S \subseteq V\}$ are the set of vertices and edges, respectively. A bijection $\rho: X \rightarrow Y$, where $X, Y \subset V(\mathcal{H})$ and $X \cap Y=\emptyset$ is a pairing on the hypergraph $\mathcal{H}$. An $(x, \rho(x))$ pair blocks an $A \in E(\mathcal{H})$ edge, if $A$ contains both elements of the pair. If the pairs of $\rho$ block all edges, we say that $\rho$ is a good pairing of $\mathcal{H}$.

Pairings are one way of showing that Breaker has a winning strategy in positional games. A good pairing $\rho$ for a hypergraph $\mathcal{H}$ can be turned into a winning strategy for Breaker in the M-B game on $\mathcal{H}$ : following $\rho$ on $\mathcal{H}$ in a M-B game, for every $x \in X$ element chosen by Maker, Breaker chooses $\rho(x)$ or in the case of $x \in Y$ vice versa (if $x \notin X \cup Y$, then Breaker can choose an arbitrary vertex). Hence Breaker can block all edges and win the game.

Since in our paper $k$-in-a-row type games play an important role, we will define $\mathcal{H}_{k}$, the hypergraph of the $k$-in-a-row games.

Definition 1. The vertices of the $\boldsymbol{k}$-in-a-row hypergraph $\mathcal{H}_{k}$ are the squares of an infinite (chess)board, i. e., an infinite square grid. The edges of the hypergraph $\mathcal{H}_{k}$ are the $k$-element sets of consecutive squares in a row horizontally, vertically or diagonally. We shall refer to the infinite rows as lines.

In the previous section (see Figure 1) we saw the first example of a Breaker winning pairing strategy i. e., a good pairing for $\mathcal{H}_{9}$. A counting type proposition indicated that there is no good pairing strategy for $\mathcal{H}_{k}$, if $k<9$. We will use this well-known proposition; hence we formulate and prove the exact statement here.

For a hypergraph $\mathcal{H}$ let $d_{2}(\mathcal{H})$ (briefly $d_{2}$ ) be the greatest number of edges that can be blocked by two vertices of $\mathcal{H}$, i. e., $d_{2}$ is the maximal co-degree of $\mathcal{H}$.

Proposition 1. If there is a good pairing $\rho$ for the hypergraph $\mathcal{H}=(V, E)$, then $d_{2}|X| / 2 \geq|\mathcal{G}|$ must hold for all $X \subset V$, where $\mathcal{G}=\{A: A \in E, A \subset X\}$.

Proof. [4] We will refer to $X$ as a sub-board. The edges of $\mathcal{G}$ can be blocked only by pairs coming from $X$. There are at most $|X| / 2$ such pairs of $\rho$ on the sub-board of size $|X|$. A pair blocks at most $d_{2}$ edges, while $|X| / 2$ pairs block at most $d_{2}|X| / 2$. So, if there are more edges on the sub-board, there can not be a good pairing. 
With the help of Proposition 1, we may conclude that there is no pairing strategy for $\mathcal{H}_{k}$ if $k<9$. In the hypergraph $\mathcal{H}_{k}, d_{2}=k-1$ because a pair blocks at most $k-1$ edges and this happens if and only if the pair is a domino. If $X$ is an $n \times n$ sub-board for sufficiently large $n$, then $|\mathcal{G}|=4 n^{2}+O(n)$ because four edges start from a square (a vertical, a horizontal and two diagonal, except at the border). By Proposition 1 , we get $(k-1) n^{2} / 2 \geq 4 n^{2}+O(n)$; that is, $k \geq 9+O(1 / n)$. One can even compute $O(n)$ exactly: $O(n)=-6(k-1) n+2(k-1)^{2}$.
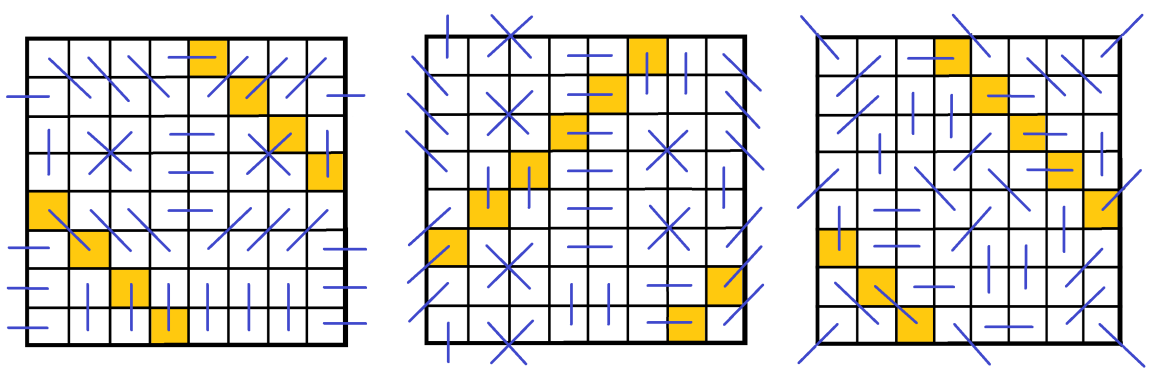

Figure 2: Some good pairings for the 9-in-a-row and some diagonal torus lines

For $\mathcal{H}_{9}$ there are some other good pairings besides the Hales-Jewett pairing, three of which can be seen in Figure 2. One can check that their extensions - by just repeating the $8 \times 8$ squares on the infinite grid - are good pairings for $\mathcal{H}_{9}$.

\subsection{Conditions for good pairings of $\mathcal{H}_{9}$}

Consider an $n \times n$ square sub-board of the infinite board. Proposition 1 tells us that $(k-1) n^{2} / 2 \geq 4 n^{2}+O(n)$, which implies that $k \geq 9+O(1 / n)$. This suggests that one must use the pairs "optimally" to block $\mathcal{H}_{9}$; that is, a pair should block the maximum possible edges of $\mathcal{H}_{9}$. We will now define the notion of optimality more precisely as follows.

Definition 2. A pairing is optimal if

1. Every pair blocks exactly $k-1$ edges.

2. There are no overblockings, i. e., every edge is blocked by exactly one pair.

3. There is no empty square, i. e., every square is contained in a pair.

Observation 1. Let us consider an optimal good pairing for $\mathcal{H}_{9}$. Then this pairing is a domino pairing in which the dominoes follow each other by 8-periodicity in each line and all squares are covered by a pair.

Proof. The first condition in Definition 2 implies that the pairing is a domino pairing, while the second gives the 8-periodicity since otherwise it would cause either overblocking or result in an unblocked edge. The lack of empty squares is just a repetition of the third condition. 
Definition 3. We call a square of a pairing anomaly in the cases where either the 8-periodicity is violated or a non-domino pair appears or there is an empty square in the pairing.

The above-mentioned pairings are anomaly-free pairings. However, according to Proposition 1 there might be $O(n)$ anomalies even in a good pairing of $\mathcal{H}_{9}$. But this conjecture was shown to be false (see Section 6 of [6]), so all good pairings for $\mathcal{H}_{9}$ are anomaly-free.

Definition 4. A pairing of the infinite board is $\boldsymbol{k}$-toric if it is a repetition of a $k \times k$ square, where $k$ is the smallest possible value.

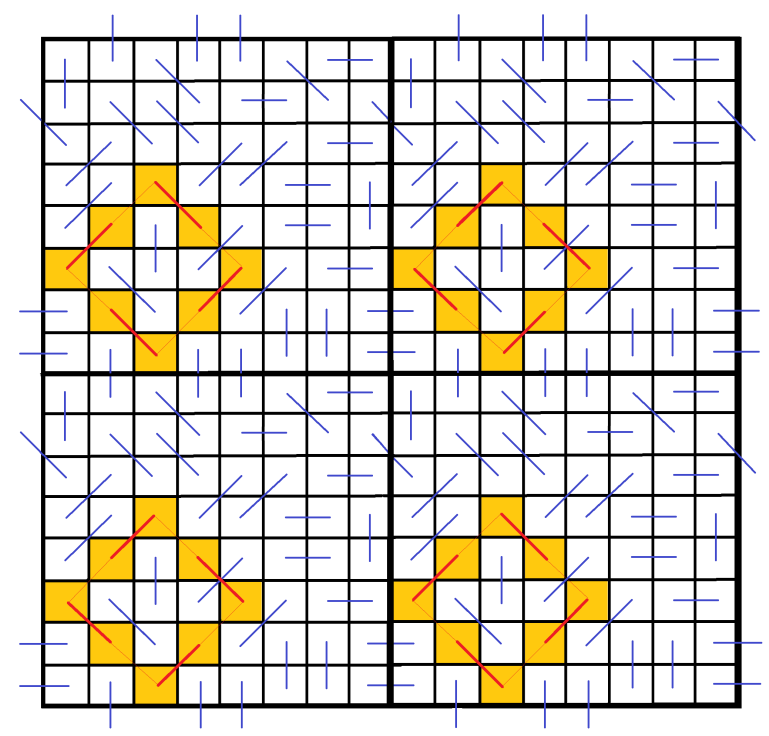

Figure 3: A 16-toric good pairing

Theorem 6 in [6] states that all good pairings for $\mathcal{H}_{9}$ are either 8-toric or 16toric. The Hales-Jewett pairing in Figure 1 and the three pairings in Figure 2 are all 8-toric. We can see a 16-toric (but not 8-toric) good pairing in Figure 3. The four $8 \times 8$ squares differ from each other only in the colored squares, where bold pairs denote the actual pairs and thin ones denote the difference. Note that considering the bottom-left (which is the same as the upper-right) or the bottomright (upper-left) $8 \times 8$ squares separately in Figure 3 we get two different good 8 -toric pairings. In [6], Corollary 8 states that all 16 -toric good pairings of $\mathcal{H}_{9}$ are just a combination of two (or more) different 8-toric ones.

In the remaining part of the study, we will describe the 8-toric good pairings for $\mathcal{H}_{9}$. 


\section{Torus game}

Let us now commence with by a new M-B game that is closely related to the 9-in-a-row game.

Definition 5. The $8 \times 8$ Maker-Breaker torus game is played on the 64 squares of the discrete torus, where there are 32 winning sets, namely the eight rows and columns and the diagonal torus lines of slope \pm 1 . We will call $\mathcal{T}_{8}$ the hypergraph of that game.

In Figure 2, we can see some diagonal torus lines marked in yellow.

Remark 1. In the case of $\mathcal{T}_{8}$, there are 32 winning sets and 64 squares on which there may be 32 pairs. Hence, if we consider the four directions (horizontal, vertical and two diagonals), then the case of $k=8$ is the unique value of $k$ for which there may be good pairings in such a way that all winning sets contain exactly one pair.

Observation 2. An arbitrary good 8-toric pairing for $\mathcal{H}_{9}$ provides a good pairing for $\mathcal{T}_{8}$.

Proof. To identify the squares of an $8 \times 8$ torus, we fix one square of the torus and call it $a 1$, and then we use the usual chess notation. Choose a good 8-toric pairing of $\mathcal{H}_{9}$ and cut an $8 \times 8$ square from that. Due to the 8 -toric condition, a 8-long line either contains a domino pair (e. g. e $4-e 5)$ or there are two half pairs in the square (e. g. $e 1$ and $e 8)$, which are neighbors on the torus.

Remark 2. The reverse is not true, because the $8 \times 8$ torus has good pairings which are not domino types. What is more, there are (non-domino) pairs which can block more than one edge (e. g. $a 4-d 8$ blocks a slope +1 and a -1 line), which allows us unpaired squares. However, by choosing only domino pairs we can always extend a good pairing of the torus to an 8-toric pairing of $\mathcal{H}_{9}$.

Remark 3. There are (only 16-toric) pairings which are good for $\mathcal{H}_{9}$, but on an $8 \times 8$ section of it the given pairing is not necessarily good for $\mathcal{T}_{8}$ (e. g. the central $8 \times 8$ section in Figure 3 ). There are some (non-domino) pairings which are good for $\mathcal{T}_{8}$, but their extensions are not good for $\mathcal{H}_{9}$. And all 8-toric, domino pairings good for $\mathcal{H}_{9}$ are also good domino pairings for $\mathcal{T}_{8}$ as well, and vice versa.

To find all the good domino pairings for the $8 \times 8$ torus is a finite task, which is not hard using a computer. However, one has to check the torus symmetries to list the non-isomorphic pairings, which makes solving the problem difficult. Next, we will investigate the difficulties and present our searching program.

\section{Generating the possible pairings on an $8 \times 8$ board}

The goal was to write a program that can find all possible pairing strategies of the infinite board for the 9-in-a-row game. Since these strategies may be constructed 
from 8 - or 16 -toric pairings and both the 8 - and the 16 -toric pairings may be deduced from pairings on an $8 \times 8$ section of the board, we looked at the $8 \times 8$ pairings only (with the toric expansion in mind).

We store a pairing in an $8 \times 8$ table, each cell representing the actual pair of the cell according to the 8 possible pairs: 0 means East, 1 South-East, etc., 7 NorthEast. Naturally, if a cell's pair is on the Eastside, then its pair has its own pair on the Westside, i. e., we fill the table two cells at a time. The algorithm itself is the usual backtracking algorithm, namely we find possible pairs for the next cell in the table having no pair so far, try all those by recursively calling the table filling function. While checking whether a pair is possible, we also make sure that there can be no overblocking, so we keep track of the blocked edges. A detailed example can be found on a webpage [8].

From previous experience, we know that speed is crucial. There are too many such pairings, so we try to reduce the number of cases. We consider two pairing strategies on the infinite board the same, if they can be transformed into each other by a translation, mirroring and rotation. So, in order not to find the same pairing several times, we apply all transformations for any pairing found on the $8 \times 8$ table. From these transformed pairings we select the smallest in the lexicographical order. This also means that such a pairing must start with 0 and 4 in the first row of the $8 \times 8$ table, so we can also reduce the number of cases by starting the table with these two numbers.

Naturally, we keep in mind the fact that the $8 \times 8$ table is expanded in an 8 toric way to the whole infinite board, while applying these transformations. More precisely:

1. We either mirror or not (2 possible cases) the table about the vertical line between columns 4 and 5 .

2. We rotate the table by $0,90,180$, and 270 degrees (4 possible cases).

3. We try all toric (that is, modulo 8) translations, which results in a table starting with a $0-4$ pair.

We select the lexicographycally smallest table as a representative for the actual pairing.

The method above reduced the number of all pairing checked to $6,210,560$ and the program found the 194,543 different pairings in about 4 minutes on a desktop computer with a $3.2 \mathrm{GHz}$ Core i7 processor using $12 \mathrm{Mb}$ of memory. The pairings themselves can be downloaded from a webpage [8]. Interestingly, the number of different pairings turns out to be a prime number.

Since we have many such different pairings, an obvious way of finding a structure is to store the pairings in a graph. Next, we will present a natural method for finding connections between pairings. 


\section{Creating a graph of "connected pairings"}

When trying to find pairings by hand one may observe that one can move a pair along the blocked edge by one step to create a new pairing using the following method:

1. Move the first pair on the table. This move creates a cell (say $A$ ) without a pair, and another cell (say $B$ ) with two pairs.

2. Move the pair containing cell $B$ which was not the previously moved pair so that cell $B$ has one pair after the move. But then another cell may have two pairs.

3. Repeat step 2 as long as it creates a cell with two pairs.

4. This method will terminate when the last move creates a new pair for cell $A$, which had no pair before the move.

Naturally, we must not forget that we are on an 8-toric pairing and we must move the pairs accordingly. Since we are on a finite table, this method will either end at step 4, or create a repeating cycle. But the latter case is not possible. Note that cell A cannot be part of the cycle, as it has no pair, and it would break the repetition. The first move that is entered in the cycle creates a hole "behind" (outside the cycle), and when the cycle comes to the same cell, the pair will move backwards, and the cycle does not begin again.

Also, it is not hard to see that we get an optimal pairing with this method. Since the original pairing was optimal, moving a pair (in an 8-toric way) along the blocked edge keeps that direction blocked. Since the method above ends in step 4 , there are no cells without a pair. We also move the pairs on a torus, so no overblocking is possible.
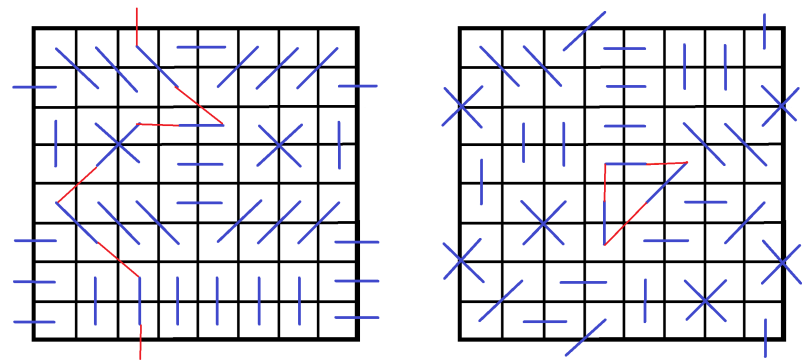

Figure 4: Two examples of connections between pairings

We say that two pairings are connected, if one can obtain the second pairing from the first one via this method (of course, we consider only different pairings as defined in the previous section). This relation is symmetric, meaning that moving 
back the last pair of the above method brings us back to the first pairing from the second one. This creates a graph where the vertices are the pairings and the edges are defined by this moving transition. Figure 4 shows two examples for this moving transition. In both cases, the first pairing contains only blue pairs, and the red dominoes show the transition to the other pairing. After computing all possible different pairings, our program readily constructs this graph. It tries to move all pairings (by trying to free up each cell in the $8 \times 8$ board), and uses the method described in the previous section to find the lexicographycally smallest representative for the new pairing. It takes about 1 minute to finish this task on the same hardware as that described above.

In the next section, we will investigate the properties of the graph we obtained.

\section{Analyzing the graph}

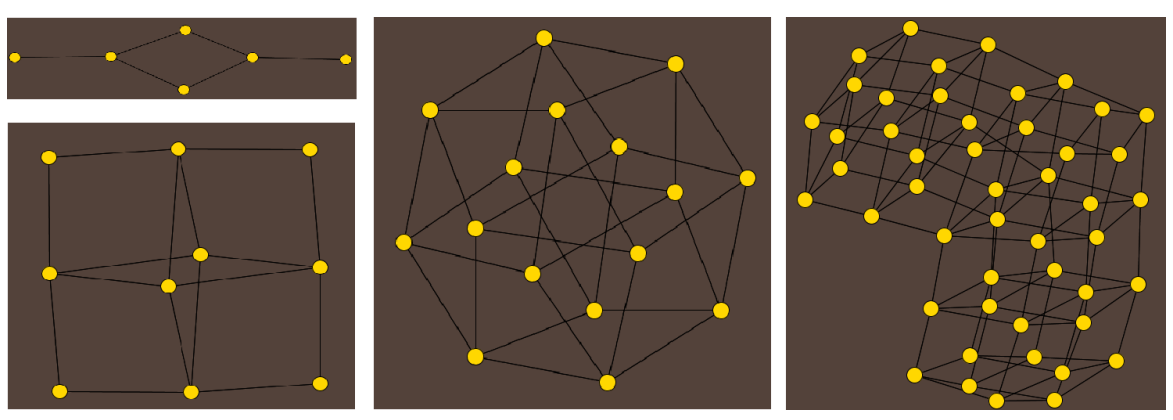

Figure 5: Some components of the given graph

Table 1: Basic parameters of the constructed graph

\begin{tabular}{|c|c|c|c|c|c|}
\hline vertices & edges & \#components & max degree & min degree & avg. degree \\
\hline 194543 & 532107 & 14 & 11 & 1 & 5.47 \\
\hline
\end{tabular}

Table 2: Degree distribution of the graph

\begin{tabular}{|c|c|c|c|c|c|c|c|c|c|c|}
\hline 1 & 2 & 3 & 4 & 5 & 6 & 7 & 8 & 9 & 10 & 11 \\
\hline 17 & 392 & 395 & 39811 & 66185 & 53222 & 25309 & 7547 & 1472 & 183 & 10 \\
\hline
\end{tabular}

The basic parameters of the above graph can be seen in Table 1. It has 194,543 vertices and 532,107 edges. The graph is not connected, which means that we cannot reach an arbitrary pairing from another by repeating the moving transition described previously. One of the 14 components of the graph is a giant component containing almost all the vertices $(194,333)$. The diameter of this component is 34 , which tells us that even this giant component does not seem to be a "smallworld" network. There are 5-5 smaller components of 10 and 16 vertices and 1-1 
components of size $6,26,48$. Note that the components containing 16 vertices are the net of a 4-dimensional cube. In Figure 5, there are some small components.

The graph is triangle-free and the length of all induced cycles is four. The degree distribution of the graph can be seen in Table 2 above. The average degree is 5.47, meaning that there are 5.47 transitions on average from a pairing to other pairings. Two examples for 1-degree vertices are in the smallest component of the graph pictured on the top left of Figure 5. We will show this component in detail.
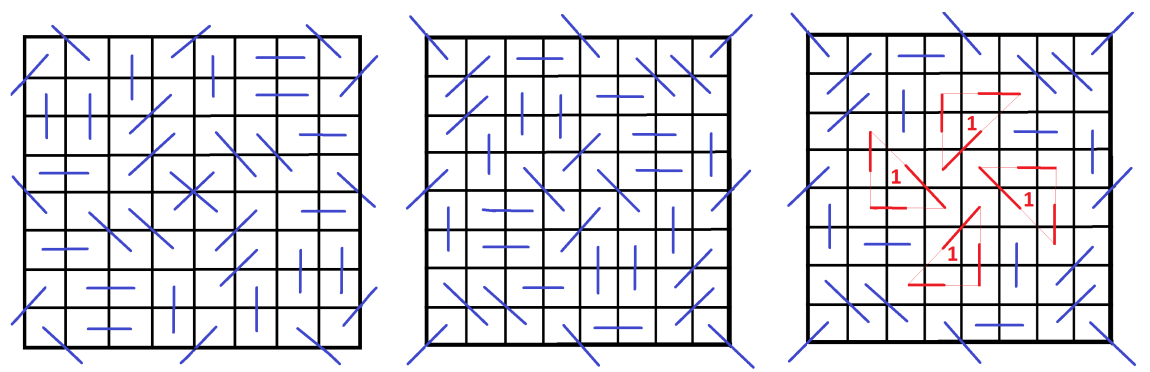

Figure 6: The same pairing twice and its four cycles

In Figure 6, we can see the same pairings on the left and middle (indicating that identifying two identical pairings is not always easy). We can see that this pairing has a rotational symmetry as well. On the right hand side of Figure 6, we can see the same pairing with its four 3-long cycles marked in red, whose cycles have two cases. Either there is a domino right to the right angle of the triangle (shown in bold in the picture, state 1) or left to the right angle (represented by thin dominoes, state 2). Note that if we change one or more cycles from state 1 to state 2 or vice versa, then we obtain another (different) good pairing. Furthermore, if we apply the moving transition described above to an arbitrary domino (not only in the cycles), then only the three dominoes in exactly one of the four cycles change.

Because of the rotational symmetry, there are six states of that group of pairings: All cycles are in state 1 ; one in state 2 the others in state 1 ; two adjacent ones are in state 1 and the other two in state 2; two non-adjacent dominoes are in state 1 and the others in state2; three are in state 2 and all four are in state 2.

It is not hard to check that the small component with six vertices is produced by these six (slightly) different pairings.

\section{The case of the hexagonal board}

Now we will examine the $k$-in-a-row Maker-Breaker game on a hexagonal board, which is the same as on a square grid with only one diagonal direction. Then Proposition 1 states that there cannot be pairing strategies if $k \leq 7$. For $k=7$ there are good pairings, as the next theorem states. All pairings are 6 -toric, which means that selecting the $6 \times 6$ torus game on a square grid with three directions 
(vertical, horizontal and slope -1 diagonal) Breaker can apply the good pairings of the 7 -in-a-row in this game as well.

Theorem 3. There exists a good pairing of the 7-in-a-row game on a hexagonal board or on a square grid with three directions (vertical, horizontal and e. g. slope -1 diagonal).

Proof. One can check that the pairing in Figure 7 is a good pairing.
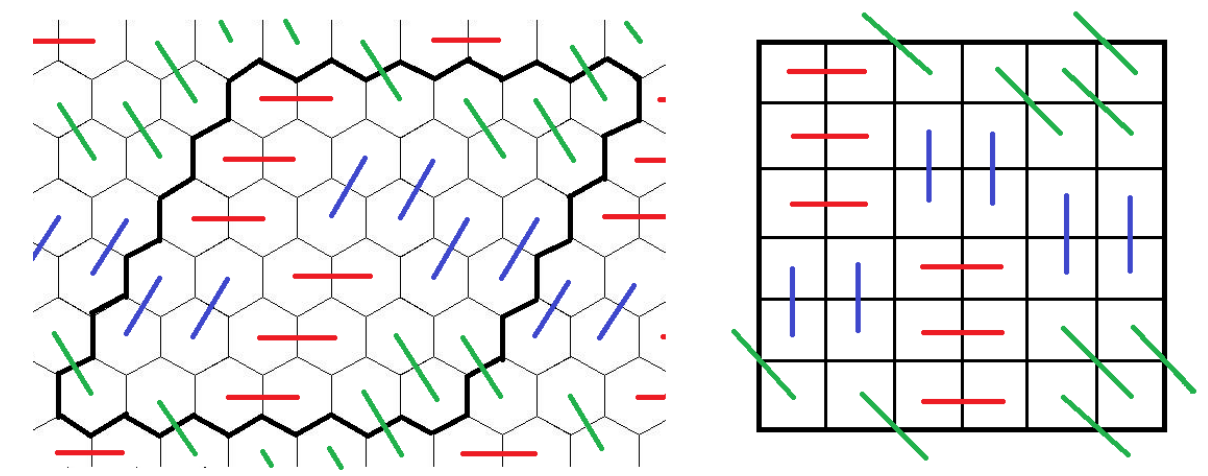

Figure 7: A good pairing for the 7-in-a-row

Using a modified version of the earlier program, we find that there exists 26 different good pairings for the 7-in-a-row game on a hexagonal board. The symmetries are different from the case of a rectangular board, because here the three directions are equivalent. Hence, there are only 6 -toric pairings and there are three rotational symmetries $(0,120,240)$ and three reflectional symmetries on the hexagonal grid.

In Figure 8, we can see the graph got in a similar way as that in Section 6. Because of the symmetries, it contains triangles. The graph has 26 vertices and 53 edges. The average degree is 4.08 and the minimal and maximal degrees are two and six. The graph is connected and its diameter is seven.

\section{Summary}

In this study, we investigated the 9-in-a-row Maker-Breaker positional game and focused on pairing strategies which guarantee that Breaker wins. We found all of the different 8-toric pairing strategies using a computer program. The main concepts of the program were also described. In order to find a structure of the 194,543 pairings, we arranged them into a graph where the vertices are the pairings themselves and the edges are the moving transitions of pairs. After our analysis of this graph, we turned to the hexagonal 7-in-a-row game and provided a smaller (instead of 194,543 only 26 vertices) and more transparent example for the pairing strategies of the $k$-in-a-row type games. 


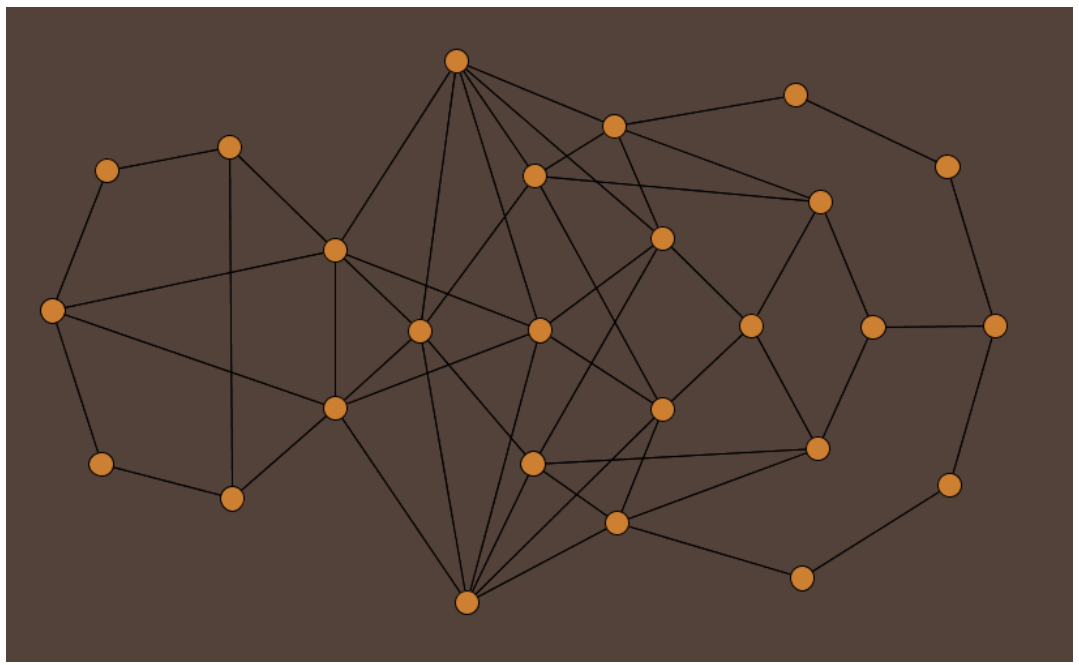

Figure 8: The graph of the 7-in-a-row game on a hexagonal board.

\section{References}

[1] L. V. Allis, H. J. van den Herik and M. P. Huntjens. Go-Moku solved by new search techniques. Proc. 1993 AAAI Fall Symp. on Games: Planning and Learning, AAAI Press Tech. Report FS93-02, pp. 1-9, Menlo Park, CA.

[2] J. Beck. Combinatorial Games, Tic-Tac-Toe Theory. Cambridge University Press, 2008.

[3] E. R. Berlekamp, J. H. Conway and R. K. Guy. Winning Ways for your mathematical plays, Volume 3, 2nd Edition. A. K. Peters, MA, 2003.

[4] A. Csernenszky, R. Martin and A. Pluhár. On the Complexity of ChooserPicker Positional Games. Integers 11, 2011.

[5] R. K. Guy and J. L. Selfridge, Problem S.10, Amer. Math. Monthly 86 (1979); solution T.G.L. Zetters 87 (1980) 575-576.

[6] L. Győrffy, G. Makay and A. Pluhár. Pairing strategies for the 9-in-a-row game. Submitted, 2016.

http: //www . math.u-szeged.hu/ lgyorffy/predok/9_pairings.pdf downloaded: 01. 09. 2016.

[7] A. W. Hales and R. I. Jewett. Regularity and positional games. Trans. Amer. Math. Soc. 106 (1963) 222-229; M.R. \# 1265.

[8] G. Makay. Personal homepage http: //www.math.u-szeged.hu/ ${ }^{\sim m a k a y / a m o b a / ~ d o w n l o a d e d: ~ 06 . ~ 04 . ~} 2016$. 